\title{
Image-guided navigation and video-assisted thoracoscopic spine surgery: the second generation
}

\author{
J. Patrick Johnson, M.D., ${ }^{1,3}$ Doniel Drazin, M.D., ${ }^{1}$ Wesley A. King, M.D., ${ }^{1}$ \\ and Terrence T. KIM, M.D.2 \\ Departments of ${ }^{1}$ Neurosurgery and ${ }^{2}$ Orthopaedics, Cedars-Sinai Medical Center, Los Angeles; and \\ ${ }^{3}$ Department of Neurosurgery, UC Davis Medical Center, Sacramento, California
}

Object. Video-assisted thoracoscopic surgery (VATS) has evolved for treatment of a variety of spinal disorders. Early incorporation with image-guided surgery (IGS) was challenged due to reproducibility and adaptability, limiting the procedure's acceptance. In the present study, the authors report their experience with second-generation IGS and VATS technologies for anterior thoracic minimally invasive spinal (MIS) procedures.

Methods. The surgical procedure is described in detail including operating room set-up, patient positioning (a lateral decubitus position), placement of the spinal reference frame and portal, radiographic localization, registration, surgical instruments, and the image-guided thoracoscopic discectomy.

Results. Combined IGS and VATS procedures were successfully performed and assisted in anatomical localization in 14 patients. The mean patient age was 59 years (range 32-73 years). Disc herniation pathology represented the most common indication for surgery ( $\mathrm{n}=8$ patients); intrathoracic spinal tumors were present in 4 patients and the remaining patients had infection and ossification of the posterior longitudinal ligament. All patients required chest tube drainage postoperatively, and all but 1 patient had drainage discontinued the following day. The only complication was a seroma that was presumed to be due to steroid therapy for postoperative weakness. At the final follow-up, 11 of the patients were improved neurologically, 2 patients had baseline neurological status, and the 1 patient with postoperative weakness was able to ambulate, albeit with an assistive device.

The evolution of thoracoscopic surgical procedures occurring over 20 years is presented, including their limitations. The combination of VATS and IGS technologies is discussed including their safety and the importance of 3D imaging. In cases of large open thoracotomy procedures, surgeries require difficult, extensive, and invasive access through the chest cavity; using a MIS procedure can potentially eliminate many of the complications and morbidities associated with large open procedures. The authors report their experience with thoracic spinal surgeries that involved MIS procedures and the new technologies.

Conclusions. The most significant advance in IGS procedures has resulted from intraoperative CT scanning and automatic registration with the IGS workstation. Image guidance can be used in conjunction with VATS techniques for thoracic discectomy, spinal tumors, infection, and ossification of the posterior longitudinal ligament. The authors' initial experience has revealed this technique to be useful and potentially applicable to other MIS procedures. (http://thejns.org/doi/abs/10.3171/2014.1.FOCUS13532)

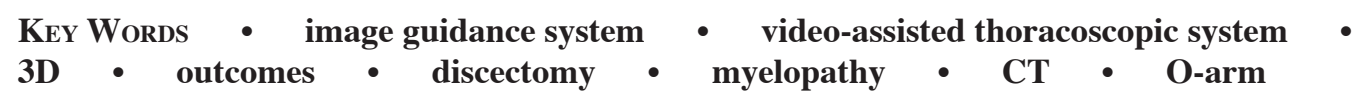

$\mathrm{V}$ IDEO-ASSISTED thoracoscopic surgery (VATS) for treatment of spinal disorders was first reported in 1993. ${ }^{12,19,20,26}$ The procedure attracted great interest as a prototype for minimally invasive spinal (MIS) surgery for thoracic disc disease but had several disadvantages. It was an unfamiliar procedure, for an infrequent pathology; there were technical difficulties with

\footnotetext{
Abbreviations used in this paper: $\mathrm{iCT}=$ intraoperative CT; IGS $=$ image-guided surgery; IRF = instrument reference frame; MIS = minimally invasive spinal; OPLL $=$ ossification of the posterior longitudinal ligament; VATS = video-assisted thoracoscopic surgery.
}

early instrumentation and surgeons had difficulty with spatial and 3D orientation on a 2D monitor. ${ }^{1,4,6,8,11,15-18}$ To overcome these challenges, we pursued the concept of incorporating image-guided surgery (IGS) into VATS. ${ }^{13,14,24}$ In 2005, we published our experience with first-generation IGS technology, for which, in addition to visual feedback from the video camera, we were also able to add fluoroscopic imaging to help surgeons navigate in the deep thoracic cavity with more familiarity. ${ }^{14}$ Unfortunately, the process of calibrating equipment required a point-matching registration procedure on the anterior surface of the spine to achieve registration of the IGS system 
with the spinal anatomy. This combination of IGS and VATS did not gain widespread acceptance, and few other centers adopted this technology. ${ }^{9,10,23}$

Frameless stereotactic IGS for spinal procedures entered a new era, or second generation, in 2006 when the $\mathrm{O}$-arm (Medtronic, Inc.) was introduced and intraoperative CT (iCT) became available for practical applications. This created a tremendous expansion of IGS procedures for posterior spinal instrumentation surgery and has become commonplace at many institutions worldwide today..$^{27-29}$

In this study, we report our experience with secondgeneration IGS and VATS technologies for anterior thoracic MIS procedures.

\section{Methods \\ Operating Room Set-Up \\ The operating room set-up for navigated minimally invasive VATS requires provisions and considerations for several specialty procedures that are done in sequence in the same operating room, some portions of which are per- formed simultaneously (Fig. 1). These procedures include the following: 1) thoracic endoscopy, 2) iCT image acqui-}

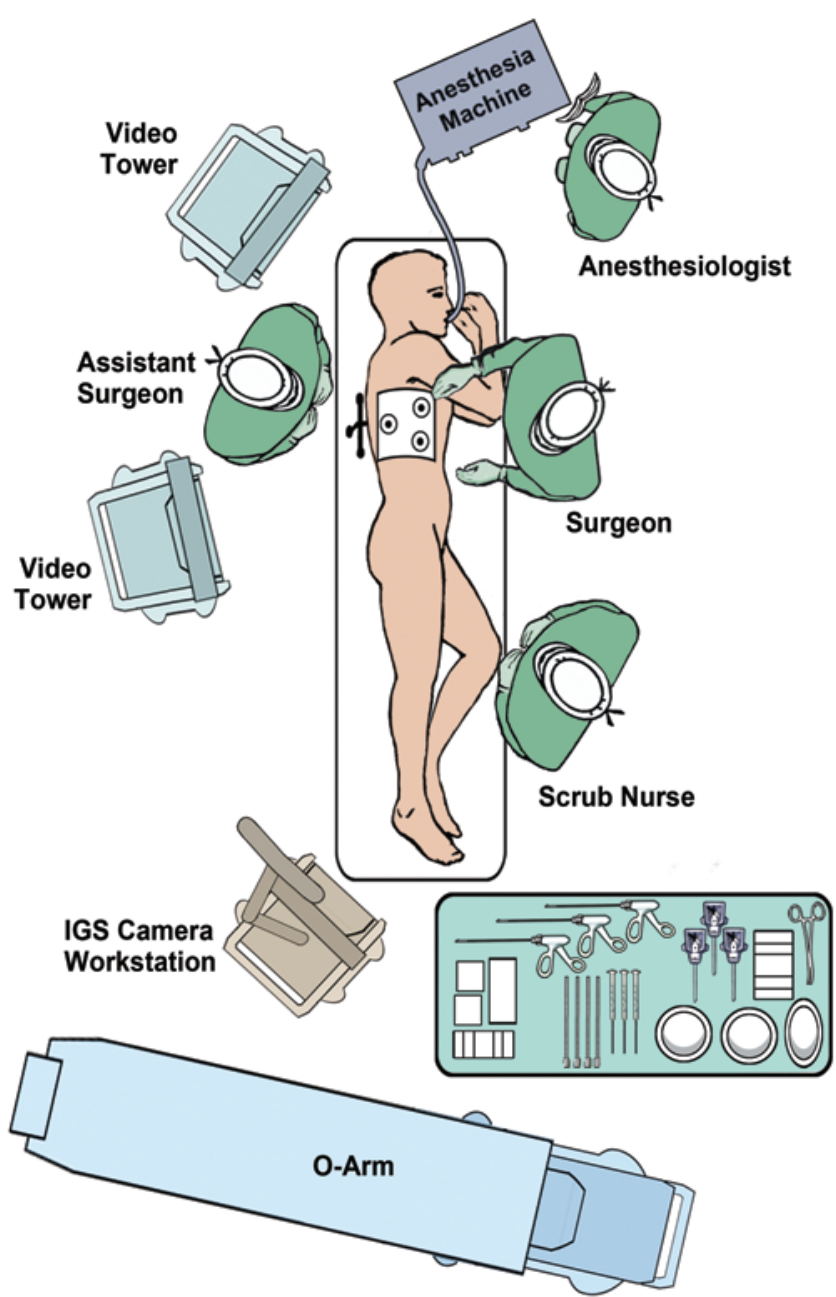

Fig. 1. Operating room set-up for the image-guided thoracoscopic procedure. Copyright Patricia Vetter. Published with permission. sition, and 3) IGS with a stereotactic navigation system to effectively guide the surgeon throughout the procedure. Unlike other spinal procedures, the complexity and the integration of these various components are uncommon, and extensive preplanning must be considered.

\section{Patient Preparation and Positioning}

Thoracoscopic spinal procedures are well described in the literature. ${ }^{5,24}$ They require induction of general anesthesia and insertion of a double lumen endotracheal tube for selective ventilation of the lung opposite the side of the procedure. The patient is secured on a radiolucent Jackson fluoroscopic operating room table in a lateral decubitus position with the operative side up (Fig. 1) and the spinal column close to the posterior edge of the table. The patient's shoulders are extended, elbows flexed to allow exposure to the lateral chest wall, and arms are carefully adducted close to the confines of the head. This important positioning of the arms allows the patient to fit onto the frame of the Jackson table and also allows the O-arm to slide cranial enough to image the surgical site without hindrance.

\section{Radiographic Localization}

The surgical level of a specific spinal lesion that is to be operated on must be determined with certainty. Traditionally, this has been done with fluoroscopic localization as described by Oskouian et al., ${ }^{24}$ prior to skin preparation and draping, and reconfirmed fluoroscopically after exposure of the spinal column and a needle marker placed in the disc space. Alternatively, localization can be confirmed with a fluoroscopic anteroposterior/lateral scout image obtained from the mobile iCT scanner. This is more likely done if the surgical thoracic level is identifiable by a distinctive radiographic appearance (that is, lytic/blastic lesion, distinct vertebral osteophytes, calcified disc herniation, and so on), which can be confirmed on a preoperative CT scan. It is always recommended that a preoperative CT scan be obtained for patients with any thoracic pathology and who are anticipated to undergo resection. In the particular case of a calcified thoracic disc herniation identified on preoperative CT, fluoroscopic localization is not required because the calcified disc can be localized with the iCT scan obtained for the IGS procedure. The patient is then prepared and draped in the standard fashion to provide exposure for the incision for both the portal placement in the posterior and anterior axillary lines and the spinal reference frame placement in the region of the spinal pathology.

\section{Placement of Spinal Reference Frame}

The spinal level and portal sites are provisionally estimated anatomically and remain the same as described in the previous publication about first-generation VATS. ${ }^{14}$ For the spinal reference frame in ISG, a midline posterior spinal incision is made at the level of the surgical site (Fig. 2 ). The spinous process of the desired level is exposed, and a bone clamp with the spinal reference frame device is attached securely to the spinous process. The first step is completed as the computer navigation requires a spinal 


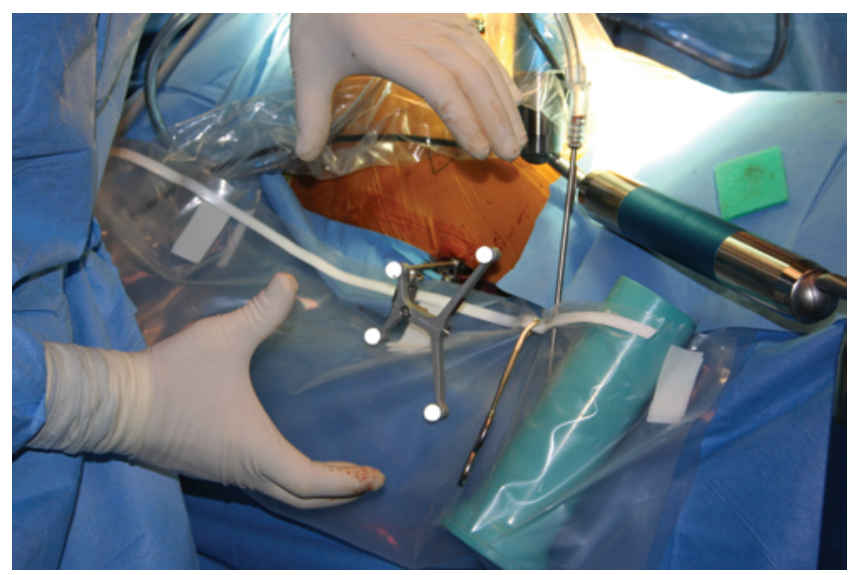

FIG. 2. Photograph showing the spinal reference frame attached to a thoracic spinous process.

reference frame for automatic registration of the acquired iCT images.

\section{Registration}

The CT images that are acquired using the O-arm system are transferred to the computer Treon S7 StealthStation (Medtronic, Inc.), and automatic registration of the CT images is carried out by the software installed in the StealthStation. The 3D data set is then verified with the standard IGS probe. The axial, coronal, and sagittal views are most commonly used with the IGS system, but the surgeon can also select other views. The most common "custom" view is a line-of-sight probe trajectory view. With the IGS navigation system, custom use of other instruments can be easily adapted with the removable instrument reference frame (IRF), described by Kim et al. ${ }^{16-18}$ An IRF can be attached to essentially any surgical instrument and used during the operative procedure. We commonly attach and calibrate the IRF to a high-speed drill (see Fig. 4), which allows the navigated drill to be used for key portions of the procedure. The IRF is an essential tool for tracking in real time during the actual drilling through the vertebral body near the spinal canal. Occasionally, a longer version of the standard reference probe may be needed for a thoracoscopic procedure because of the long distance from the chest wall to the spinal column in patients with a larger chest cavity.

\section{Portal Placement}

The endoscopic portal placement is arranged in a triangular fashion with the endoscope portal in the posterior axillary line that is directly perpendicular to the level of the spinal pathology (Fig. 3). A pneumatic-arm endoscope holder can be used to maintain position of the endoscope during the procedure. The two working ports are placed in the anterior axillary line slightly rostral and caudal to the operative level of interest.

\section{Surgical Instruments}

The instruments used for a thoracoscopic spinal procedure differ only in that they are a longer version of essentially the same instruments used for an open opera-
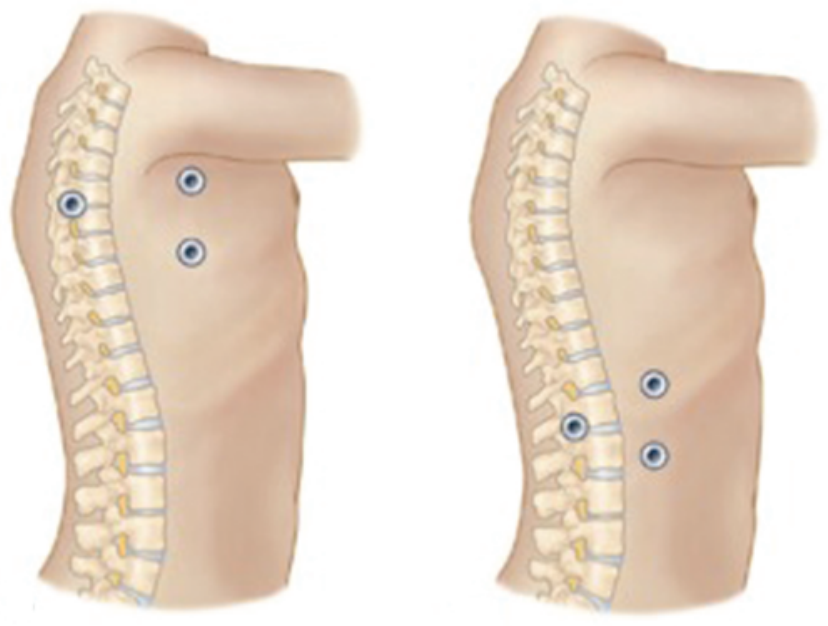

FIG. 3. Portal placement for the thoracoscopic IGS procedure, with posterior portal located perpendicular to the surgical level at T5-6 (left) and T12-L1 (right). Copyright Patricia Vetter. Published with permission.

tion. The surgeon typically uses a long suction device in the nondominant hand and a similar but longer version of the procedural instruments (monopolar or bipolar cautery, high-speed drill, curette, Kerrison rongeur, and so on) in the dominant hand. The high-speed drill (Fig. 4) is usually the key instrument in a thoracoscopic disc procedure; tracking the drill with a reference frame attached provides a real-time tracking of the drill along its trajectory and the drill bit during the procedure.

\section{Image-Guided Thoracoscopic Discectomy Procedure}

The intrathoracic portion of the procedure begins with retraction of the lung anteriorly; the operating table can be rotated slightly to allow the lung to fall anteriorly. The rib head is overlying the disc of interest in all but the lower levels (where the rib only articulates with the corresponding vertebra). Once identified, the parietal pleura is opened with either a monopolar cautery or a harmonic scalpel (Ethicon, Inc.) over the rib head and anteriorly to expose the anulus of the disc space. The segmental vessels can usually be preserved as they cross at the midportion of the vertebral bodies. If necessary, they can be coagulated and divided with the harmonic scalpel or other cautery device.

The high-speed drill, adapted with an IRF for tracking, is used to drill out and remove the rib head (Fig. 5) and expose the lateral aspect of the pedicle. As drilling proceeds across the vertebral body in the posterior aspect of the disc space, the high-speed drill is continuously tracked visually with the surgical endoscope video monitor and simultaneously with the IGS system on the StealthStation monitor. The drilling is just anterior to the spinal cord at the level of the disc space. Image guidance allows the surgeon to determine initial trajectory and drill tip position relative to the spinal canal during the entire operative procedure. The trajectory of vertebral body resection is visualized with image guidance to guide the surgeon across to the contralateral pedicle (Fig. 6). When the decompression is completed, the posterior longitudinal ligament is opened, 


\section{J. P. Johnson et al.}

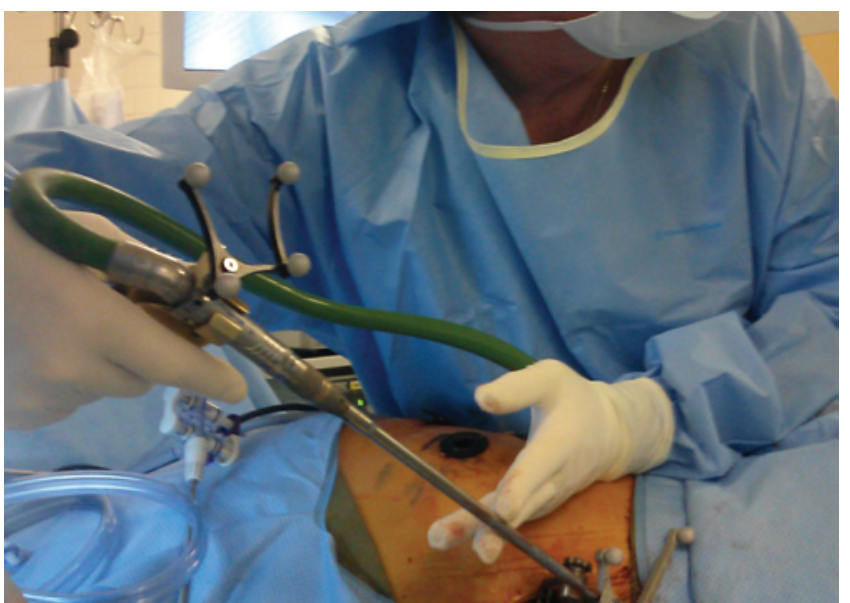

FIG. 4. High-speed drill with the IRF attached and being registered as an "image-guided" surgical instrument.

allowing removal of any disc material that may have extruded into the canal. This allows adequacy of the decompression to be assessed at the end of the procedure with the IGS probe. The procedure is then completed by achieving bony hemostasis with bone wax; epidural hemostasis is achieved with Gelfoam (Pharmacia Corp.). A small chest tube is inserted through one of the endoscope portals; once placement is confirmed endoscopically into the apex of the chest cavity, the lung is reinflated by the anesthesiologist. The endoscopic ports are removed, the incisions are closed in layers, and sterile dressings are then applied. The chest tube is placed to a closed suction system in the operating room and converted to water seal in the recovery room after a chest radiograph confirms that the lung is inflated. The chest tube can usually be removed within 24-48 hours.

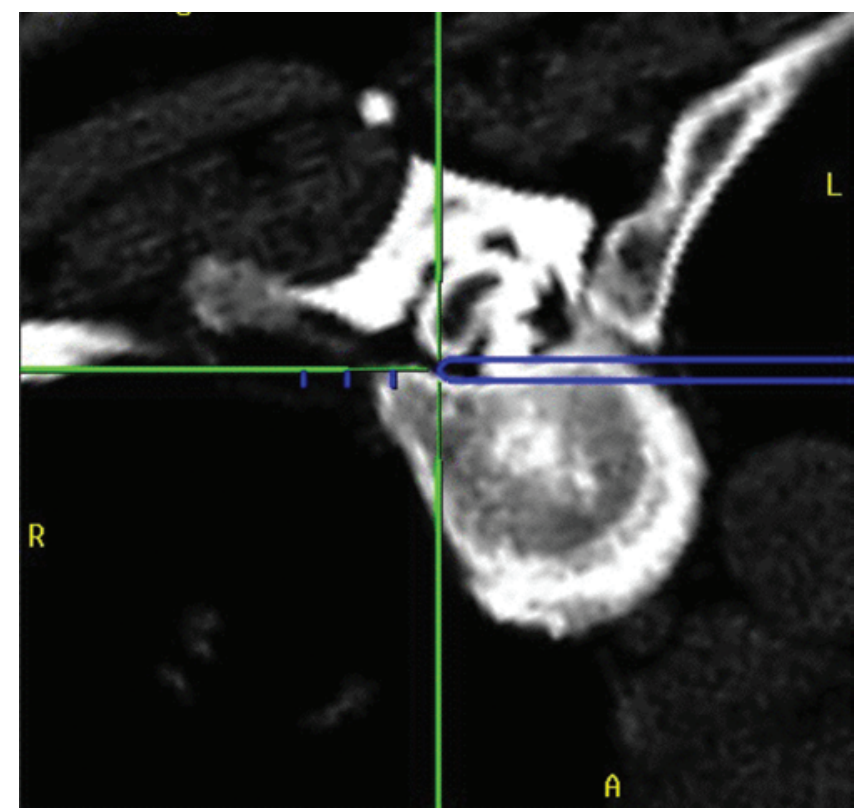

FIG. 5. Surgical trajectory of the IGS high-speed drill for initial steps of rib head removal to expose the pedicle and continue the procedure. $A=$ anterior; $L=$ left; $R=$ right.

\section{Results}

\section{Patient Demographics}

The combined second-generation CT-IGS and MIS VATS procedures were successfully performed in 14 patients (6 women and 8 men). The mean age of the patients was 59 years (range 32-73 years). Patient characteristics and demographics are listed in Table 1.

Of the 8 patients who underwent surgery for symptomatic disc herniation, 6 patients had a single-level treatment and 2 patients had a 2-level treatment. All patients with symptomatic disc herniation had presenting symptoms of progressive myelopathy (Fig. 7). Clinical pathology demonstrated a calcified (6 patients) disc herniation in all the patients with single-level herniations and soft disc herniations in all 2 patients with 2-level herniations. All disc herniation patients had midline disc lesions that typically, and otherwise, required open thoracotomy and could not be easily treated with a posterior or posterolateral surgical approach.

Of the patients who underwent surgery with a combined VATS and IGS to remove an intrathoracic spinal tumor, 3 had nerve sheath tumors (that is, schwannoma) that were primarily extraforaminal in location (Fig. 8), and 1 patient had a hemorrhagic intraspinal hemangioma. Three patients underwent open posterior intraspinal tumor resection performed in conjunction with an IGS and VATS procedure. ${ }^{7}$ One patient underwent surgery for ossification of the posterior longitudinal ligament (OPLL) extending over 3 levels and 1 patient underwent surgery for osteomyelitis. The mean estimated blood loss was 550 $\mathrm{ml}$ (range 100-2000 $\mathrm{ml}$ ), and the mean operating room time was 300 minutes (range 200-500 minutes).

All patients required chest tube drainage postoperatively, and all but 1 patient had it discontinued the following day after radiographic confirmation of the absence of significant pneumothorax, hemothorax, or pleural effusion. One patient needed an extra day of chest tube drainage because of a multiple-day admission in the intensive care unit. Of the 14 patients who underwent surgery, 3 required a postoperative admission to the intensive care unit for monitoring. One patient had immediate postoperative leg weakness that was treated with steroid therapy (previous spinal cord injury guidelines), while the other 2 patients were observed overnight (for shortness of breath) and were transferred out the following day. The average length of stay was 4.75 days (range 2-7 days). The patient who received steroid therapy was a 72 -year-old man with multiple medical comorbidities in whom a postoperative symptomatic seroma developed that was subsequently drained and successfully treated with antibiotics. One patient required cardiac monitoring postoperatively for exacerbation of a preexisting atrial fibrillation. Twelve of the patients were discharged to home, whereas 2 were transferred to the inpatient rehabilitation unit. Of the 2 admitted to the rehabilitation unit, 1 patient had weakness and the other required assistance with balance and gait that has since been restored. Half of the patients experienced chest wall paresthesia postoperatively, but only 1 patient described the paresthesia as severe at last followup. The mean follow-up period was 9 months (range 3-36 


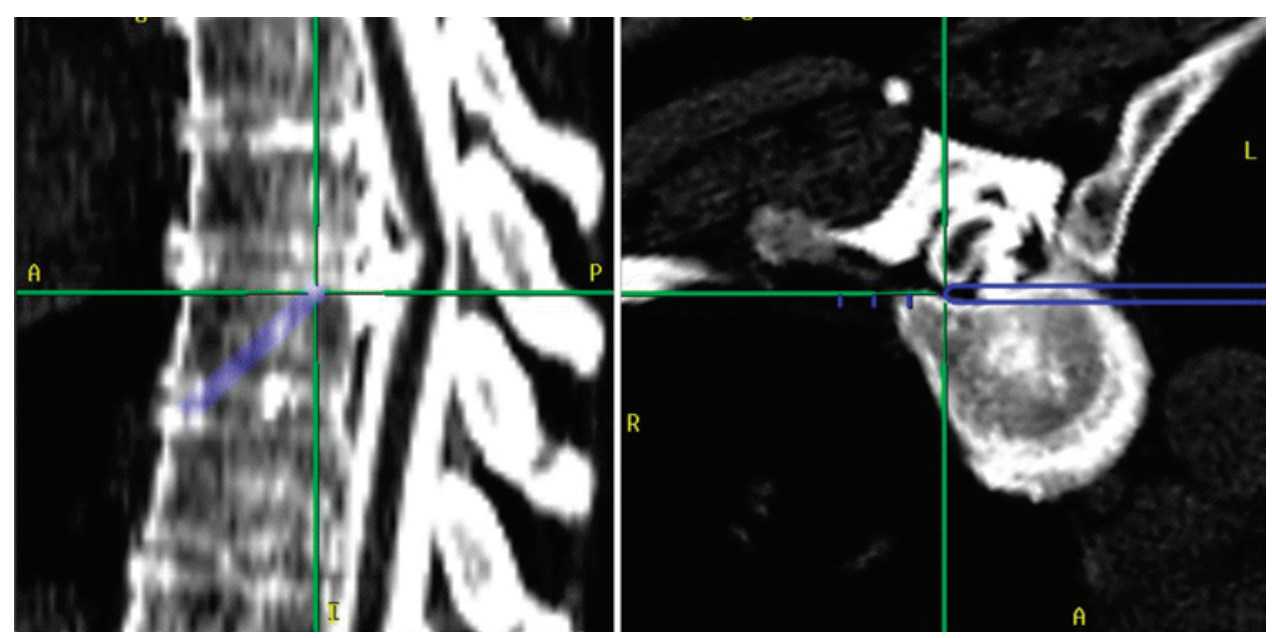

FIG. 6. Sagittal (left) and axial (right) IGS views of surgical trajectories of the decompression as the drill is advanced across to the contralateral pedicle. $P=$ posterior.

months). At final follow-up, 11 of the patients were improved neurologically (specifically, less gait disturbance and less pain), 2 patients were neurologically at baseline, and 1 patient with postoperative weakness was able to ambulate, albeit with a walker. There were no complications independently related to the IGS procedure.

\section{Discussion}

\section{Evolution of Thoracoscopic Spinal Surgery}

The thoracic spine can harbor many different pathological entities. The literature contains numerous articles reporting the indications, techniques, and procedures for sympathectomy, discectomy, vertebral biopsy, anterior release, vertebral corpectomy, internal fixation, and

TABLE 1: Summary of the preoperative demographics of patients undergoing IGS and VATS*

\begin{tabular}{cc}
\hline Characteristic & Value \\
\hline no. of patients & 14 \\
sex (\%) & \\
men & $8(57)$ \\
women & $6(43)$ \\
age in yrs & \\
mean & 59 \\
range & $32-73$ \\
preop diagnosis (\%) & \\
herniated disc & $8(57)$ \\
1 level & $6(75)$ \\
2 level & $2(25)$ \\
tumor & $4(28)$ \\
schwannoma & $3(75)$ \\
hemangioma & $1(25)$ \\
infection & $1(7)$ \\
OPLL & $1(7)$ \\
\hline
\end{tabular}

\footnotetext{
* Values represent the number (\%) of patients unless otherwise specified.
}

tumor resection. The advantages of thoracoscopy over thoracotomy have been well documented and include shorter hospitalization, decreased operative blood loss, and shortened operative time (after the surgeon's learning curve). ${ }^{19,26}$ Thoracoscopic spinal procedures were developed 20 years ago by surgeons in Germany and the United States who were intrigued by MIS techniques that were an alternative to large, open thoracotomy procedures. ${ }^{20,25,26}$ Although several different minithoracotomy procedures have also been developed and described as MIS techniques, the VATS procedure remained the only closed minimally invasive procedure for the thoracic spine in which video-assisted technology is used., ${ }^{1,21}$

Although VATS initially generated great interest, it was technically unfamiliar to most surgeons, was required in only a small number of patients, and was rarely performed. Therefore, only a limited number of spine surgeons mastered and regularly performed VATS, largely due to the technical challenges of a complex spinal procedure being performed immediately adjacent to the spinal cord, when inadequate spatial orientation was available due to $2 \mathrm{D}$ imaging during a surgical procedure requiring an understanding of the complex 3D anatomy.

\section{Previous Limitations of Thoracoscopic Spinal Surgery and} First-Generation Image Guidance

Thoracoscopic procedures with the VATS technique were associated with a steep learning curve because they used unfamiliar endoscopic procedures and they used 2D imaging when $3 \mathrm{D}$ orientation is critical to performing the procedure. ${ }^{1}$ Perhaps the most important reason that a 3D understanding is needed in the surgical field is the need for appropriate spatial orientation for safety and effectiveness of the surgery. The depth-of-field was improved with graduated instruments that facilitated some of the limitations but did not resolve them.

With regard to first-generation image-guidance technology, IGS required that the surgeon either 1) obtain a CT scan with the patient under general anesthesia or 2) use historical CT data and do a manual point-to-point registration. ${ }^{2,3,5,13,14}$ These early registration protocols required 

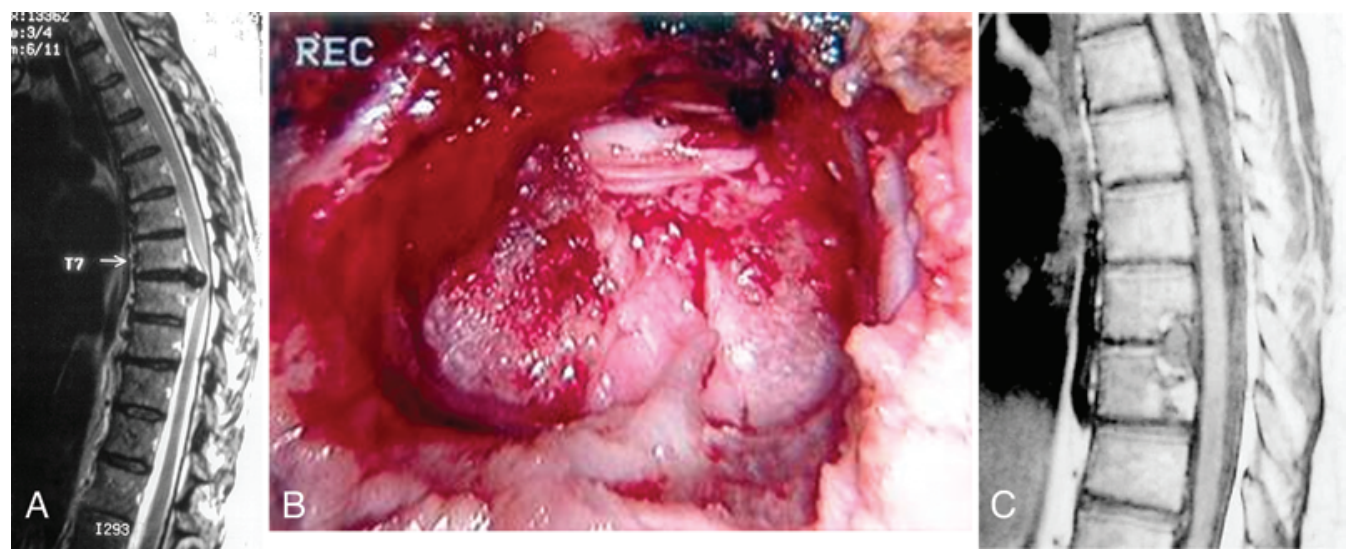

Fig. 7. A: Preoperative T2-weighted sagittal MR image of a large thoracic disc herniation. B: Postsurgical decompression of a thoracic disc herniation. C: T1-weighted sagittal MR image acquired after the combined IGS and VATS decompression procedure.

matching 3 distinct radiographic points on the axial CT scans with 3 anatomical points on the spinal column. ${ }^{14}$ In the case of VATS, the major limitation was the inability to efficiently register the preoperative CT scan to the anterior spinal column because of the lack of distinct anatomical contours that could be accurately identified. These factors limited the clinical use of thoracoscopy and VATS for many procedures.

Treatment of thoracic disc disorders with a combination of VATS and IGS has advanced significantly since the initial case report in $20010^{2,3}$ In their early feasibility study, Assaker et al. ${ }^{3}$ reported on obtaining a CT scan in a single patient under general anesthesia; an IRF was attached to the spinal column, and registration was thereby completed for the surgical procedure. As indicated in the publication, the procedure was successful; however, the multistep process of IRF attachment and transport of the patient to the radiology department and from the operating room for image acquisition was logistically problematic and precluded routine clinical use. In 2005, we published the first clinical series on the practical application of image guidance in which VATS and IGS technologies were combined. ${ }^{14}$ For these procedures, we obtained a preoperative CT scan, which used an intraoperative anterior spinal registration that was technically difficult to reproduce and unreliable.

\section{VATS and Second-Generation IGS Spinal Procedures}

Although VATS technology has not significantly advanced in applications in the thoracic spine, the two most significant advancements in second-generation IGS procedures resulted from the development of mobile iCT scanners and automatic registration with the IGS workstation. Current IGS procedures begin with obtaining an iCT scan when an IRF device is either 1) attached to the spinous process with a clamp or 2) fixed to an immediately adjacent structure such as a Mayfield head holder or pelvic fixation pin.

Once the CT scanning process is completed, the images are immediately transferred to a computer workstation where software renders a 3D reconstruction, automatically registers the images in relation to the IRF and spine, and eliminates the logistics that affected IGS procedural accuracy. The factors now determining accuracy of the IGS system include the following: 1) the inherent technical accuracy of the IGS system, 2) the registration process by the IGS system, 3) the slice thickness of the scan data, and 4) any intraoperative events affecting the IRF that may result in accuracy errors. The key part of this last factor is based on the IRF device remaining undisturbed. In recent years, many investigators have described applications for image guidance in essentially any region of the spine for posterior instrumentation and some anterior procedures. ${ }^{17,22,29,30}$

The accuracy of the combined IGS and VATS allows the surgeon to establish an appropriate trajectory of the surgical procedure during bone drilling, the extent of
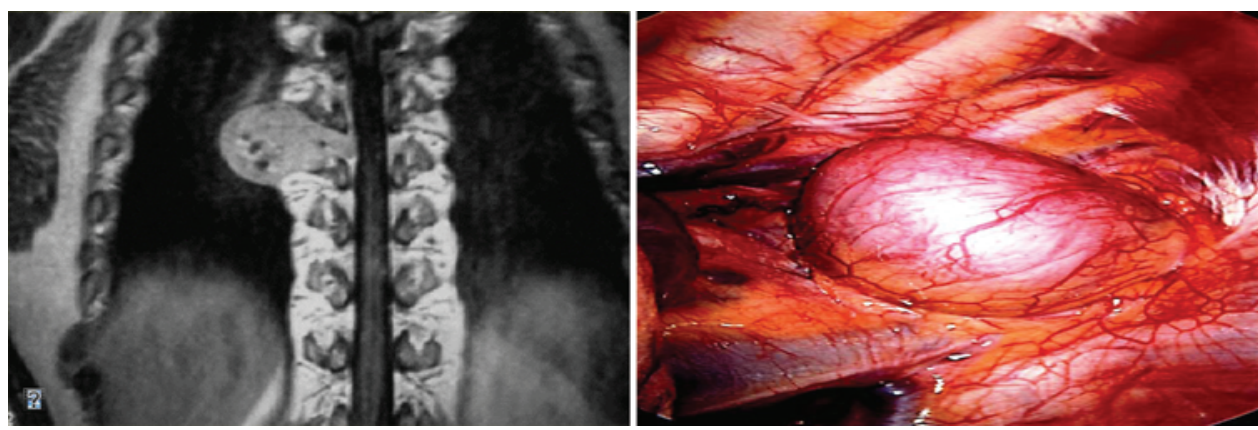

FIG. 8. Left: Coronal T1-weighted enhanced MR image showing a thoracic schwannoma extending into the foramen but not into the spinal canal. Right: Intraoperative image. The tumor can be resected entirely with an IGS and VATS procedure. 
bone resection, the depth of decompression during a disc herniation procedure, and the proximity to the foramen and spinal canal during a nerve sheath tumor resection. Although the experience of the senior author has been that 3D spatial orientation in the IGS has improved safety, decreased operating time, and improved workflow, the scope of the present study did not include scientific qualification of these parameters.

This paper reports our institutional experience in which thoracic spinal pathologies (symptomatic disc herniations, tumors, infection, and OPLL) were preferentially treated using MIS procedures with IGS and VATS technologies. If treated with large open thoracotomy procedures, these surgeries would have required difficult, extensive, and invasive access through the chest cavity. Using IGS and VATS to treat disc herniations and spine tumors can potentially eliminate many of the complications and morbidities associated with large invasive procedures. The inherent limitation of this paper is that it reports only our current institutional experience. The data, however, are consistent with our prior publication wherein we compared thoracoscopic and open thoracotomy cases. ${ }^{13}$ We surmise that the combination of advanced experience with thoracoscopy, efficient workflow, and faster automated registration may explain improved intraoperative factors. This report establishes that thoracic spinal pathologies, like disc herniations and spinal tumors, can be safely treated using MIS VATS and newest-generation IGS technologies.

\section{Conclusions}

The most significant advance in IGS procedures has resulted from $\mathrm{iCT}$ scanning and automatic registration with the IGS workstation. In MIS surgery, the 3D technology provides spatial orientation of the complex anatomy, which we believe will improve the accuracy, safety, and effectiveness of spinal surgery. Our initial experience has revealed that image guidance can be successfully used in conjunction with VATS techniques for thoracic discectomy and spinal tumors and is potentially applicable to a variety of other MIS procedures. Future large-scale, multicenter studies in patients with different underlying pathologies is needed to evaluate clinically relevant outcomes.

\section{Disclosure}

Dr. Kim reports being a consultant for DePuy Synthes.

Author contributions to the study and manuscript preparation include the following. Conception and design: Johnson. Acquisition of data: Drazin. Analysis and interpretation of data: Drazin. Drafting the article: Johnson, Drazin. Critically revising the article: Johnson, Drazin, Kim. Reviewed submitted version of manuscript: all authors. Approved the final version of the manuscript on behalf of all authors: Johnson.

\section{References}

1. Anand N, Regan JJ: Video-assisted thoracoscopic surgery for thoracic disc disease: classification and outcome study of 100 consecutive cases with a 2-year minimum follow-up period. Spine (Phila Pa 1976) 27:871-879, 2002

2. Assaker R, Cinquin P, Cotten A, Lejeune JP: Image-guided endoscopic spine surgery: Part I. A feasibility study. Spine (Phila Pa 1976) 26:1705-1710, 2001

3. Assaker R, Reyns N, Pertruzon B, Lejeune JP: Image-guided endoscopic spine surgery: Part II: clinical applications. Spine (Phila Pa 1976) 26:1711-1718, 2001

4. Bolger C, Wigfield C: Image-guided surgery: applications to the cervical and thoracic spine and a review of the first 120 procedures. J Neurosurg 92 (2 Suppl): 175-180, 2000

5. Faciszewski T, Winter RB, Lonstein JE, Denis F, Johnson L: The surgical and medical perioperative complications of anterior spinal fusion surgery in the thoracic and lumbar spine in adults. A review of 1223 procedures. Spine (Phila Pa 1976) 20:1592-1599, 1995

6. Foley KT, Smith MM: Image-guided spine surgery. Neurosurg Clin N Am 7:171-186, 1996

7. Ghostine S, Vaynman S, Schoeb JS, Cambron H, King WA, Samudrala S, et al: Image-guided thoracoscopic resection of thoracic dumbbell nerve sheath tumors. Neurosurgery 70: 461-468, 2012

8. Glossop ND, Hu RW, Randle JA: Computer-aided pedicle screw placement using frameless stereotaxis. Spine (Phila Pa 1976) 21:2026-2034, 1996

9. Haberland N, Ebmeier K, Grunewald JP, Hliscs R, Kalff RL: Incorporation of intraoperative computerized tomography in a newly developed spinal navigation technique. Comput Aided Surg 5:18-27, 2000

10. Holly LT, Bloch O, Johnson JP: Evaluation of registration techniques for spinal image guidance. J Neurosurg Spine 4: 323-328, 2006

11. Holly LT, Bloch O, Obasi C, Johnson JP: Frameless stereotaxy for anterior spinal procedures. J Neurosurg 95 (2 Suppl): 196-201, 2001

12. Huang TJ, Hsu RW, Sum CW, Liu HP: Complications in thoracoscopic spinal surgery: a study of 90 consecutive patients. Surg Endosc 13:346-350, 1999

13. Johnson JP, Filler AG, McBride DQ: Endoscopic thoracic discectomy. Neurosurg Focus 9(4):E11, 2000

14. Johnson JP, Stokes JK, Oskouian RJ, Choi WW, King WA: Image-guided thoracoscopic spinal surgery: a merging of 2 technologies. Spine (Phila Pa 1976) 30:E572-E578, 2005

15. Kalfas IH, Kormos DW, Murphy MA, McKenzie RL, Barnett $\mathrm{GH}$, Bell GR, et al: Application of frameless stereotaxy to pedicle screw fixation of the spine. J Neurosurg 83:641-647, 1995

16. Kim KD, Johnson JP, Babbitz JD: Image-guided thoracic pedicle screw placement: a technical study in cadavers and preliminary clinical experience. Neurosurg Focus 10(2):E2, 2001

17. Kim KD, Johnson JP, Bloch BS O, Masciopinto JE: Computerassisted thoracic pedicle screw placement: an in vitro feasibility study. Spine (Phila Pa 1976) 26:360-364, 2001

18. Kim KD, Johnson JP, Masciopinto JE, Bloch O, Saracen MJ, Villablanca JP: Universal calibration of surgical instruments for spinal stereotaxy. Neurosurgery 44:173-178, 1999

19. Landreneau RJ, Hazelrigg SR, Mack MJ, Dowling RD, Burke D, Gavlick J, et al: Postoperative pain-related morbidity: video-assisted thoracic surgery versus thoracotomy. Ann Thorac Surg 56:1285-1289, 1993

20. Mack MJ, Regan JJ, Bobechko WP, Acuff TE: Application of thoracoscopy for diseases of the spine. Ann Thorac Surg 56: 736-738, 1993

21. McAfee PC, Regan JR, Zdeblick T, Zuckerman J, Picetti GD III, Heim S, et al: The incidence of complications in endoscopic anterior thoracolumbar spinal reconstructive surgery. A prospective multicenter study comprising the first 100 consecutive cases. Spine (Phila Pa 1976) 20:1624-1632, 1995

22. Nolte L, Zamorano L, Arm E, Visarius H, Jiang Z, Berlerman $\mathrm{U}$, et al: Image-guided computer-assisted spine surgery: a pilot study on pedicle screw fixation. Stereotact Funct Neurosurg 66:108-117, 1996 


\section{J. P. Johnson et al.}

23. Nolte LP, Slomczykowski MA, Berlemann U, Strauss MJ, Hofstetter R, Schlenzka D, et al: A new approach to computer-aided spine surgery: fluoroscopy-based surgical navigation. Eur Spine J 9 (Suppl 1):S78-S88, 2000

24. Oskouian RJ Jr, Johnson JP, Regan JJ: Thoracoscopic microdiscectomy. Neurosurgery 50:103-109, 2002

25. Regan JJ, Mack MJ, Picetti GD III: A comparison of video-assisted thoracoscopic surgery (VATS) with open thoracotomy in thoracic spinal surgery. Today Ther Trends 11:203-218, 1994

26. Rosenthal D, Rosenthal R, de Simone A: Removal of a protruded thoracic disc using microsurgical endoscopy. A new technique. Spine (Phila Pa 1976) 19:1087-1091, 1994

27. Tian NF, Huang QS, Zhou P, Zhou Y, Wu RK, Lou Y, et al: Pedicle screw insertion accuracy with different assisted methods: a systematic review and meta-analysis of comparative studies. Eur Spine J 20:846-859, 2011

28. Tjardes T, Shafizadeh S, Rixen D, Paffrath T, Bouillon B, Steinhausen ES, et al: Image-guided spine surgery: state of the art and future directions. Eur Spine J 19:25-45, 2010
29. Tormenti MJ, Kostov DB, Gardner PA, Kanter AS, Spiro RM, Okonkwo DO: Intraoperative computed tomography imageguided navigation for posterior thoracolumbar spinal instrumentation in spinal deformity surgery. Neurosurg Focus 28(3):E11, 2010

30. Youkilis AS, Quint DJ, McGillicuddy JE, Papadopoulos SM: Stereotactic navigation for placement of pedicle screws in the thoracic spine. Neurosurgery 48:771-779, 2001

Manuscript submitted November 19, 2013.

Accepted January 20, 2014.

Please include this information when citing this paper: DOI: 10.3171/2014.1.FOCUS13532.

Address correspondence to: J. Patrick Johnson, M.D., The Spine Center, Cedars-Sinai Medical Center,444 S. San Vicente Blvd., Mark Goodson Bldg., Ste. 800, Los Angeles, CA 90048. email: patrick. johnson@cshs.org. 